\title{
A novel animal model for vulnerable atherosclerotic plaque: dehydrated ethanol lavage in the carotid artery of rabbits fed a Western diet
}

\author{
Ruochi Zhao ${ }^{1 \#}$, Hongyan Liu ${ }^{2 \#}$, Shangshi Zhang ${ }^{3}$, Qi Lu ${ }^{4}$, Xiaohong Fei ${ }^{1}$, Honglin Zhou ${ }^{1}$, Junsong Liu ${ }^{1}$, \\ Honghua Ye ${ }^{1}$, Xiaomin Chen ${ }^{1}$, Hanbin Cui ${ }^{1}$
}

${ }^{1}$ Cardiology Center, Ningbo First Hospital, Ningbo University, Ningbo, China; ${ }^{2}$ Neurology Department, Ningbo First Hospital, Ningbo University, Ningbo, China; ${ }^{3}$ Cardiovascular Department, Shangrao People's Hospital, Shangrao, China; ${ }^{4}$ Cardiovascular Department, Yinzhou People's Hospital, Ningbo University, Ningbo, China

Contributions: (I) Conception and design: H Cui, H Ye, X Chen; (II) Administrative support: None; (III) Provision of study materials or patients: X Fei, H Zhou, J Liu; (IV) Collection and assembly of data: R Zhao, H Liu, S Zhang, Q Lu; (V) Data analysis and interpretation: R Zhao, H Cui; (VI) Manuscript writing: All authors; (VII) Final approval of manuscript: All authors.

\#These authors contributed equally to this work.

Correspondence to: Hanbin Cui, MD, PhD. Cardiology Center, Ningbo First Hospital, Ningbo University, 59 Liuting Street, Haishu District, Ningbo 315010, China. Email: hbcui_nbdyyy@outlook.com.

Background: The study of unstable atherosclerotic plaques is limited by the absence of ideal animal models to reproduce the plaque instability observed in humans. In this study, we attempted to develop a novel animal model for vulnerable atherosclerotic plaques using dehydrated ethanol lavage in rabbits fed a Western diet (WD).

Methods: A total of 30 New Zealand White (NZW) rabbits were randomized to 5 groups, including a control group with or without WD, a balloon injury with WD group, and an ethanol injury with or without WD group. Operations were conducted using the right common carotid artery as the target vessel. All animals were followed up for 3 months unless a vascular event occurred. Blood samples and carotid artery specimens were ultimately collected for analysis of atherogenesis.

Results: Compared to rabbits in which lesions were induced by balloon injury, those subjected to an ethanol lavage with high cholesterol diet showed progressive atherosclerotic lesions in all carotid artery segments, which were characterized by greater plaque burden, smaller minimum lumen area (MLA), and increased vulnerability as indicated by abundant macrophages, scattered smooth muscle cell (SMC) composition, higher matrix metalloproteinase-9 (MMP-9) expression in plaques, thinner fibrous cap thickness, and higher possibility of stroke event (50\% vs. 0\%). Meanwhile, the serum interleukin-1 $($ IL-1 $\beta$ ) and monocyte chemoattractant protein-1 (MCP-1) levels in the ethanol injury group with a high-cholesterol diet were significantly higher than those in the balloon injury group after 3 months (all $\mathrm{P}<0.01$ ).

Conclusions: We successfully established a novel animal model for vulnerable atherosclerosis by ethanol exposure of the carotid segment that has a higher predictive value for the probability of ischemic events than the balloon injury model. Therefore, it may represent a promising animal model for investigating new therapeutic approaches, novel imaging modalities, and underlying mechanisms for vulnerable atherosclerotic plaque.

Keywords: Animal model; vulnerable atherosclerotic plaque; endothelial cell (EC) injury; inflammatory marker

Submitted May 06, 2021. Accepted for publication Oct 25, 2021.

doi: $10.21037 / \mathrm{cdt}-21-291$

View this article at: https://dx.doi.org/10.21037/cdt-21-291 


\section{Introduction}

Atherosclerotic cardiovascular disease, a leading cause of death and disability worldwide, is frequently associated with vulnerable atherosclerotic plaque and subsequent thrombosis (1-3). The cellular processes that lead to the characteristic histologic features of vulnerable plaques have been identified, and they are fundamentally attributed to the inflammatory cascade triggered by injury to the arterial endothelium. However, despite these advances, definitive evidence in animal models that indicates the relevance of plaque disruption in occlusive thrombus formation and subsequent cardiovascular events, is lacking (4). Moreover, new biomarkers or noninvasive molecular imaging approaches to detect vulnerable atherosclerotic plaques are needed to enable early diagnosis. Ultimately, new strategies for subduing active plaques may be essential to preventing serious clinical events triggered by disruption and erosion of the atherosclerotic plaque. For these key issues, animal experiments are indispensable tools in elucidating the mechanisms, testing new biomarkers, and exploring novel treatment strategies. However, studies of plaque activation, rupture, and thrombosis have been hampered by a paucity of ideal animal models (5), and several limitations to the existing models of plaque rupture have been noted (6). Furthermore, atherosclerotic lesions in the commonly used genetically modified mice seldom develop plaque disruption with thrombosis-a mechanism that commonly complicates the human disease (4).

Although controversy still exists concerning whether alcohol intake has a direct cardio-protective effect or provokes atherosclerosis (7), it is clear that long-term, especially high-volume, ethanol exposure provokes atherogenesis via significant induction of proinflammatory response, endothelial dysfunction, and induction of foam cell formation (8). Additionally, dehydrated ethanol is usually used for chemoablation therapy of hypertrophic obstructive cardiomyopathy (9), as it can result in the coagulated necrosis of endothelial cells (ECs) and myocardium. Cumulatively, these reasons provided us the rationale to establish an anticipant animal model of unstable atherosclerotic plaque via dehydrated ethanol lavage in the carotid artery. We therefore established a novel rabbit model of vulnerable atherosclerotic plaques using dehydrated alcohol lavage in a rabbit carotid artery segment followed by a high cholesterol diet for 3 months. The typical features of vulnerable atherosclerotic plaque were observed in the treated segment of the carotid artery. Moreover, 3 rabbits exposed to dehydrated alcohol lavage experienced stroke, and the lesions responsible for these events were documented via computed tomography angiography (CTA) and histologic examination. However, we found that advanced atherosclerotic plaque was induced by a combination of ethanol damage and a Western diet (WD) instead of transient ethanol damage alone.

We present the following article in accordance with the ARRIVE (Animal Research: Reporting of in Vivo Experiments) reporting checklist (available at https://dx.doi. org/10.21037/cdt-21-291).

\section{Methods}

\section{Preparation of atherosclerotic animal model}

Before the establishment of the animal model, we performed a pilot experiment to verify the effect of dehydrated ethanol on the viability of ECs. Human coronary artery ECs (HCAEC; ScienCell Research Laboratories, Carlsbad, CA, USA) were treated with dehydrated ethanol for 10 seconds and then washed with phosphate-buffered saline (PBS) and EC medium (SciencCell). The EC viability was evaluated via a Cell Counting Kit-8 (CCK-8) cell viability assay (Figure 1), which showed that the majority of ECs (90\%) were killed after 10 seconds by treatment with dehydrated ethanol. Based on this finding, we attempted to establish an animal model via EC damage induced by dehydrated ethanol exposure.

Then, healthy adult New Zealand White (NZW) rabbits (male, weight $2.5 \pm 0.23 \mathrm{~kg}, 4$ months of age), obtained from the Experimental Animal Center, Medical College of Ningbo university, were used to establish the model for vulnerable atherosclerosis. All animals were randomly assigned to 1 of the following groups: group A, fed a regular chow diet (normal control, $\mathrm{n}=6$ ); group $\mathrm{B}$, fed a Westerntype diet simply (cholesterol 1\% w/w and butter 6\% w/w; Medicine Bio-pharmaceutical Inc., Yangzhou, China; $n=6$ ); group $\mathrm{C}$, underwent balloon dilation injury (balloon size $2.5 \mathrm{~mm} \times 15 \mathrm{~mm}$; Medtronic Inc., Fridley, MN, USA) at a pressure of $12 \mathrm{~atm}$ for 30 seconds in the right common carotid artery $(n=6)$; group $D$, underwent a surgery of dehydrated ethanol lavage in the right common carotid artery segment $(\mathrm{n}=6)$; and group $\mathrm{E}$, underwent the same procedure as group $\mathrm{D}$, but were instead fed a regular chow diet after the surgery $(n=6)$. All animals in group $C$ and D were then fed a WD for 3 months, which was the same as the treatment for group B. 

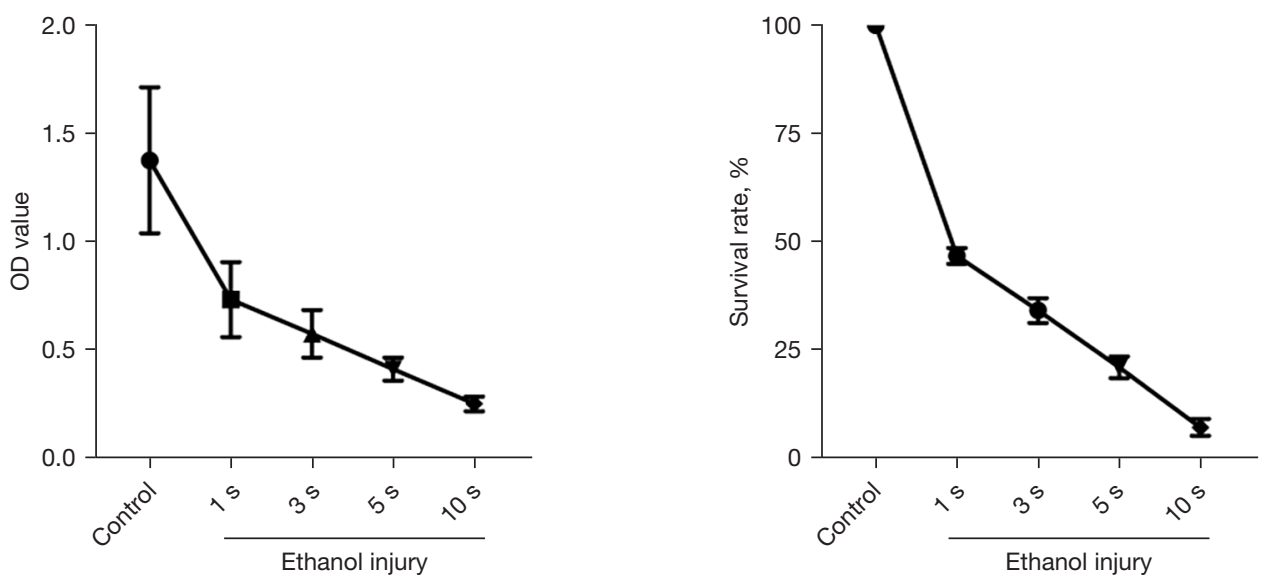

Figure 1 CCK8 assay of human coronary artery ECs. Left and right panels represent OD value and survival rate of EC, respectively, after treatment of HCAEC with dehydrated ethanol for 10 seconds. Cell proliferation was represented as cell quantity. Data are presented as mean $\pm \mathrm{SD}(\mathrm{n}=6)$. EC, endothelial cell; OD, optical density; HCAEC, human coronary artery EC.

The animals (group C, D, and E) were anesthetized using an intravenous injection of pentobarbital sodium $(30 \mathrm{mg} / \mathrm{kg})$ and were subsequently placed on a surgical table in the dorsal recumbent position. Before the surgical procedure, an intramuscular injection of benzylpenicillin (0.4 megaunit; Harbin Pharmaceutical Group Co. Ltd., Harbin, China) was administered to prevent infection. A midline incision was made on the neck, the right common carotid artery was surgically exposed, and blood flow in a segment $4 \mathrm{~cm}$ in length $(1 \mathrm{~cm}$ distal to the aorta bifurcation site) was blocked using 2 artery clamps. For the procedure of balloon injury in group $\mathrm{C}$, the proximal segment of the common carotid artery was punctured using a $24 \mathrm{G}$ needle $(0.7 \mathrm{~mm})$. Then, a percutaneous transluminal coronary angioplasty (PTCA) guide wire $(0.014$ inch $/ 0.36 \mathrm{~mm})$ was introduced into the artery via the needle. Next, a balloon catheter (size $2.5 \mathrm{~mm} \times 15 \mathrm{~mm}$; Medtronic Inc., USA) was advanced into the target segment in the right common carotid artery over the guidewire and then inflated at pressure of $12 \mathrm{~atm}$ for 30 seconds. This procedure was repeated 3 times, after which the catheter was removed. For the dehydrated ethanol lavage procedure in group $\mathrm{D}$ and $\mathrm{E}$, a needle with a $1-\mathrm{mL}$ aseptic syringe was inserted into the proximal region, blood was completely extracted, and the segment was rinsed with PBS. The target segment was then flushed with dehydrated ethanol, using a volume up to the amount of blood extracted via the puncture needle, which was kept in the vessel segment for 10 seconds. The ethanol was then completely extracted, and the vessel was again rinsed with PBS. In groups C, D, and E, the 2 artery clamps used to stop blood flow were removed when the treatment was finished, pressure was applied to the pinhole for a short time to prevent bleeding, and the surgical incision was then closed. All animals were then followed up for 3 months.

The serum lipid levels, including total cholesterol (TC), triglycerides (TG), high-density lipoprotein cholesterol (HDL-C), and low-density lipoprotein cholesterol (LDL-C) were tested by the enzymatic method at baseline and at end of the experiment, respectively. Additionally, the serum inflammatory cytokines including high-sensitivity C-reactive protein (hs-CRP; MyBioSource, Inc., San Diego, CA, USA), serum interleukin-1 $\beta$ (IL-1 $\beta ;$ R\&D systems, Shanghai, China), and monocyte chemotactic protein 1 (MCP-1; MyBioSource, Inc.) were also tested with enzymelinked immunosorbent assay (ELISA) kits at end of the experiment, respectively.

Experiments were performed under a project license (No. NBU20210023) granted by the Ethics Committee of the Experimental Animal Center, Ningbo University, in accordance with the guidelines and regulations approved by the Chinese government for the care and use of animals. A protocol was prepared before the study without registration

\section{Histological and immunobistochemical staining}

After 3 months or the occurrence of vascular events such as stroke, the animals were euthanized and their experimental vessels were harvested for atherogenesis to be analyzed. The specimens were perfusion-fixed with $10 \%$ formalin acetate for 12 hours, dehydrated, and embedded in paraffin. Finally, 
the specimens were cut into $4-\mu m$ thick serial sections. The adjacent serial cross-sections $(4 \mu \mathrm{m})$ were respectively processed in hematoxylin and eosin (HE), and cellular composition analysis, which included immunohistochemical (IHC) staining with antibodies against $\alpha$-smooth muscle cell actin ( $\alpha$-SMC, dilution 1:50; Sigma-Aldrich, St. Louis, MO, USA), von Willebrand factor (vWF, for endothelium, dilution 1:50; Thermo Fisher Scientific, Waltham, MA, USA), CD68 (for macrophages, dilution 1:100; DAKO, Santa Clara, CA, USA), and matrix metalloproteinase-9 (MMP-9, dilution 1:50; Abcam, Cambridge, UK). All tissue slides were washed with $\mathrm{PBS}$ at $\mathrm{pH}$ 7.4. Endogenous alkaline phosphatase was blocked using $3 \% \mathrm{H}_{2} \mathrm{O}_{2}$, and then rinsed with $\mathrm{PBS}$, followed by incubation with goat anti-mouse immunoglobin $\mathrm{G}(\mathrm{IgG})$ peroxidase conjugate (secondary antibody, dilution $1: 500$ ) at $37^{\circ} \mathrm{C}$ for 30 minutes. The IHC staining was visualized using an ABC kit (Fuzhou Maixin Biological Technology Development Co., Fujian, China). Nucleus counterstaining was performed using Mayer's hematoxylin. A Movat Pentachrome Stain Kit (American Master Tech.) was used to evaluate the thickness of fibrous cap and other components in the plaque. A Leica CCD camera DFC420 connected to a Leica DM IRE2 microscope (Leica Microsystems Imaging Solutions, Ltd., Cambridge, UK) was used as an imaging system. Mean density, obtained by interest optical density (IOD)/area of lesion (IOD/area), was used to determine the positivestaining density under Image-Pro Plus v. 6.0 software (Media Cybernetics, Inc., Bethesda, MD, USA).

\section{Characteristics of atherosclerotic plaque}

The atherosclerotic lesions developed in this study were classified as early fatty streaks, early and advanced fibrous plaques, and vulnerable atherosclerotic plaques. The lesion terminology used here has been previously defined (10-12). We used 4 parameters for quantitative determination of the lesions: (I) the maximal intimal thickness (MIT), which was defined as the maximal measurement from the luminal surface to the internal elastic lamina (IEL); (II) the maximal media thickness (MMT), defined as the maximal distance from the IEL to the external elastic lamina (EEL) of the vessel wall; (III) plaque burden, defined as the plaque volume normalized to the vessel volume $\times 100 \%$; and (IV) minimum lumen area (MLA), defined as the vascular area minus the plaque area. All 4 of these parameters were calculated via Image-Pro Plus v6.0 software (https://www. mediacy.com/imagepro).
Rupture-prone vulnerable plaques are typically associated with the presence of a highly inflammatory cell content and a large necrotic core (NC) covered by a thin fibrous cap, the latter being characterized by decreased smooth muscle cell (SMC) and extracellular matrix (ECM) content. Hence, cellular composition of atherosclerotic plaque including ECs, monocyte-derived macrophages, and SMC, as well the expression level of MMP-9 in plaque was measured via IHC methods, each being labeled with the aforementioned antibodies against $\alpha-S M C, v W F, C D 68$, and MMP-9, respectively. The expression levels of the proteins of interest in the atherosclerotic plaque were calculated as mentioned above, which was based on the mean density of the positive IHC staining for target proteins.

\section{Determination of carotid atherosclerotic lesions using CTA}

Under general anesthesia, the rabbits were placed in a supine position on a plastic board, and medical tape was used to minimize motion artifacts. We then performed CTA using a volume multidetector CT scanner (Aquilion ONE, Toshiba Medical Systems, Tochigi, Japan), equipped with $320 \mathrm{~mm} \times 0.5 \mathrm{~mm}$ detector rows to detect the development of carotid atherosclerotic lesions in the rabbit. A total of $10 \mathrm{~mL}$ nonionic contrast medium followed by $4 \mathrm{~mL}$ saline was infused at a rate of $0.5 \mathrm{~mL} / \mathrm{sec}$ via an earlobe vein, after which a dynamic acquisition sequence with a gantry a rotation speed of 1 rotation per second was completed. The dynamic acquisition sequence consisted of 1 mask volume $(80 \mathrm{kVp}, 200 \mathrm{mAs})$ and 22 dynamic volumes $(80 \mathrm{kVp}$, $120 \mathrm{mAs}$ ). Scanning began from the roof of the skull to the chest, covering the entire carotid artery segment of interest and the intracranial vessel. After subtracting the mask volume from the dynamic volumes, a total of 7,040 $(22 \times 320)$ images were stored in DICOM files.

\section{Statistical analysis}

Data are expressed as the mean \pm standard deviation (SD) of the different treatment groups. A nonparametric MannWhitney test was used to compare the characteristics of atherosclerotic lesions in different groups, including cellular staining density and parameters of plaque characteristics, as the data from replicates of tissues staining were not normally distributed. For the comparison of cholesterol levels and inflammatory markers, a Student's $t$-test was used to compare the differences between 2 groups, and a 1-way analysis of variance (ANOVA) with Tukey's post hoc test was 

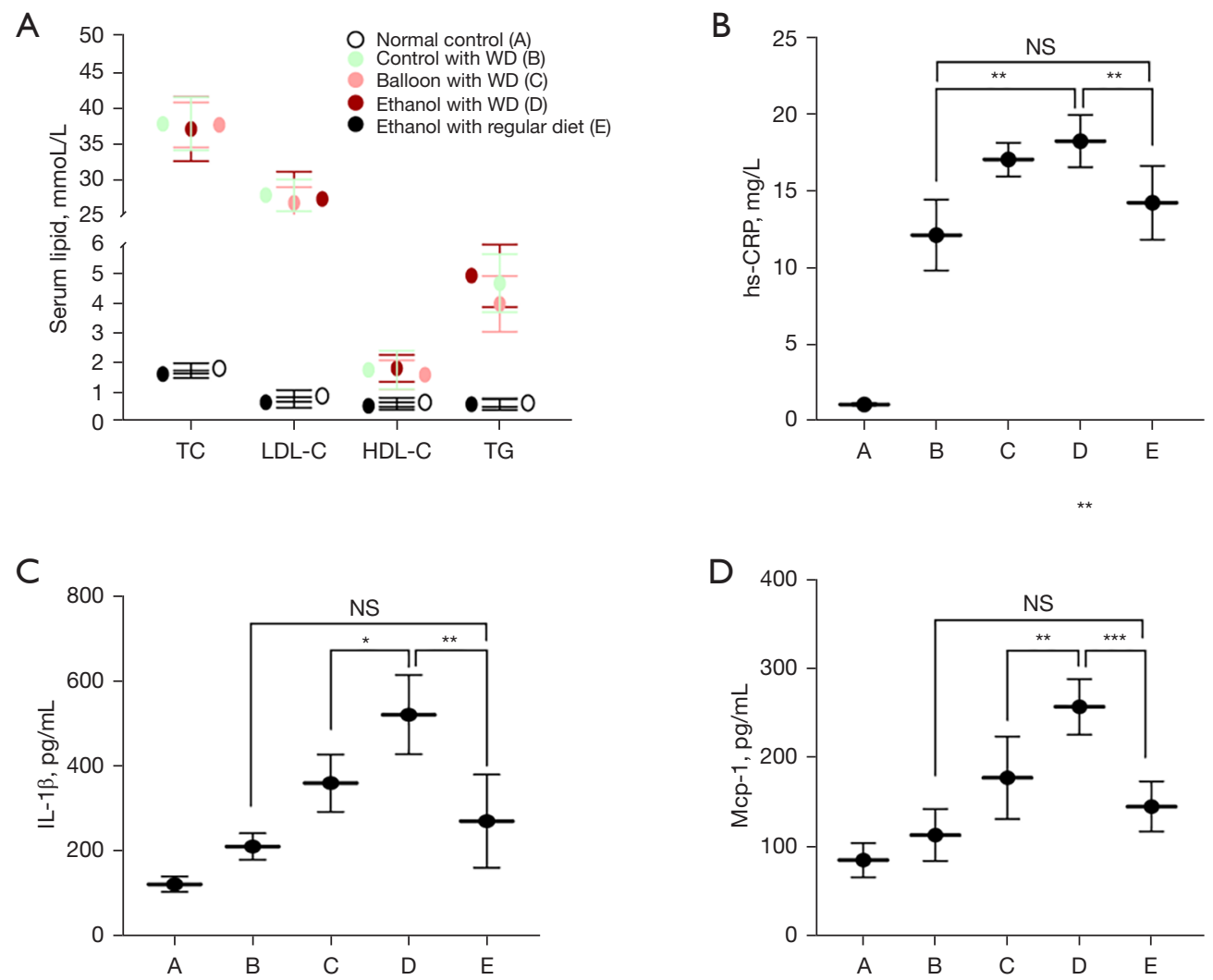

Figure 2 Serum lipid profile and inflammatory markers. (A) Serum lipid level measurements at three months, including TC, HDL-C, LDL-C and TG. Normal diet groups in figure contain group A and E, while western diet groups contain group B, C and D. Panels B, C, and D represent serum hs-CRP (B), IL-1 $\beta$ (C) and MCP-1 (D) measurements at end of observation, respectively. *, $\mathrm{P}<0.05 ;{ }^{* *}, \mathrm{P}<0.01 ;$ ***, $\mathrm{P}<0.001$ vs. normal control (group A) or counterpart group. TC, total cholesterol; HDL-C, high-density lipoprotein cholesterol; LDL-C, low-density lipoprotein cholesterol; TG, triglycerides.

used to detect differences among all the groups. A 2-sided $P$ value of $<0.05$ was considered statistically significant.

\section{Results}

\section{Procedure and manifestation}

All animals survived the procedure until they were killed, and vessel specimens were harvested after 3 months or occurrence of vascular events. The body weight of rabbits fed a WD had significantly increased compared with that of rabbits fed a regular chow diet $(2.84 \pm 0.17$ vs. $2.53 \pm 0.19 \mathrm{~kg}$; $\mathrm{P}<0.05)$, while there was no difference in body weight within groups that were fed a WD. Notably, 3 rabbits in group D (dehydrated ethanol exposure with WD) had ischemic stroke within 80 to 90 days after surgery, and the diagnosis was supported by CTA and histologic examinations (described below).

\section{Serum lipid profile and inflammatory markers}

Compared with animals fed a regular diet (group A and E), rabbits fed a WD (groups B, C, and D) had significantly increased serum lipids, including TC, LDL-C, HDL-C, and TG levels (Figure $2 A$; all $\mathrm{P}<0.01$ ). Both a high-fat diet (WD) and surgery could significantly increase hs-CRP, especially in the ethanol injury combined with a high-fat diet (group D) $(1.1 \pm 0.1 \mathrm{mg} / \mathrm{L}, 12.1 \pm 2.3 \mathrm{mg} / \mathrm{L}, 17.2 \pm 1.1 \mathrm{mg} / \mathrm{L}$, $18.2 \pm 1.7 \mathrm{mg} / \mathrm{L}$, and $14.2 \pm 2.4 \mathrm{mg} / \mathrm{L}$, in groups A to $\mathrm{E}$, respectively; $\mathrm{P}<0.01$; Figure $2 \mathrm{~B}$ ). Of note, the difference of hs-CRP between surgery groups $\mathrm{C}$ and $\mathrm{D}$, as well that between groups $\mathrm{B}$ and $\mathrm{E}$ did not reach significance (both $\mathrm{P}>0.05)$. Furthermore, both IL-1 $\beta$ (521.8 \pm 93.7 vs. $360.9 \pm 67.5 \mathrm{pg} / \mathrm{mL} ; \mathrm{P}<0.05)$ and MCP-1 levels $(257.0 \pm 31.3$ vs. $177.5 \pm 46.4 \mathrm{pg} / \mathrm{mL}, \mathrm{P}<0.01)$ increased more significantly in group $\mathrm{D}$ compared with those in group $\mathrm{C}$ (Figure $2 C, 2 D$ ). Additionally, serum IL-1 $\beta$ and MCP-1 levels between 
Table 1 Summary of atherogenesis in different groups

\begin{tabular}{|c|c|c|c|c|c|}
\hline Features of atherogenesis & $\begin{array}{l}\text { Normal control with } \\
\text { ND (Group A) }\end{array}$ & $\begin{array}{c}\text { Control with WD } \\
\text { (Group B) }\end{array}$ & $\begin{array}{l}\text { Balloon injury with } \\
\text { WD (Group C) }\end{array}$ & $\begin{array}{c}\text { Ethanol lavage with } \\
\text { WD (Group D) }\end{array}$ & $\begin{array}{c}\text { Ethanol injury with } \\
\text { normal diet (Group E) }\end{array}$ \\
\hline Incidence of fatty streak (n) & 0 & $5 / 6$ & - & - & $6 / 6$ \\
\hline Maximal intima thickness $(\mu \mathrm{m})$ & $9.9 \pm 1.3$ & $60.7 \pm 8.1^{\Delta}$ & $396.4 \pm 70.1^{\Delta \#}$ & $613.5 \pm 110.6^{\Delta \# \dagger}$ & $71.2 \pm 10.5^{\Delta}$ \\
\hline Maximal media thickness $(\mu \mathrm{m})$ & $64.7 \pm 5.4$ & $72.43 \pm 3.6$ & $139.4 \pm 24.3^{\text {*\# }}$ & $142.8 \pm 15.7^{\star \#}$ & $80.4 \pm 9.8$ \\
\hline Minimum lumen area $\left(\mathrm{mm}^{2}\right)$ & $3.80 \pm 0.72$ & $2.54 \pm 0.56^{*}$ & $1.13 \pm 0.35^{\Delta \#}$ & $0.51 \pm 0.20^{\Delta \#+}$ & $2.26 \pm 0.43^{*}$ \\
\hline
\end{tabular}

Values were represented as mean \pm SD. -, no evidence for just fatty streak in group $C$ and $D$, which probably evolve into the advanced atheroma. *, $\mathrm{P}<0.01$ and ${ }^{\triangle}, \mathrm{P}<0.001$ compared with group $\mathrm{A} ;{ }^{*}, \mathrm{P}<0.01$ compared with group $\mathrm{B}$ or $\mathrm{E} ;{ }^{\dagger}, \mathrm{P}<0.01$ compared with group $\mathrm{C}$. WD, western-type diet; ND, normal diet.
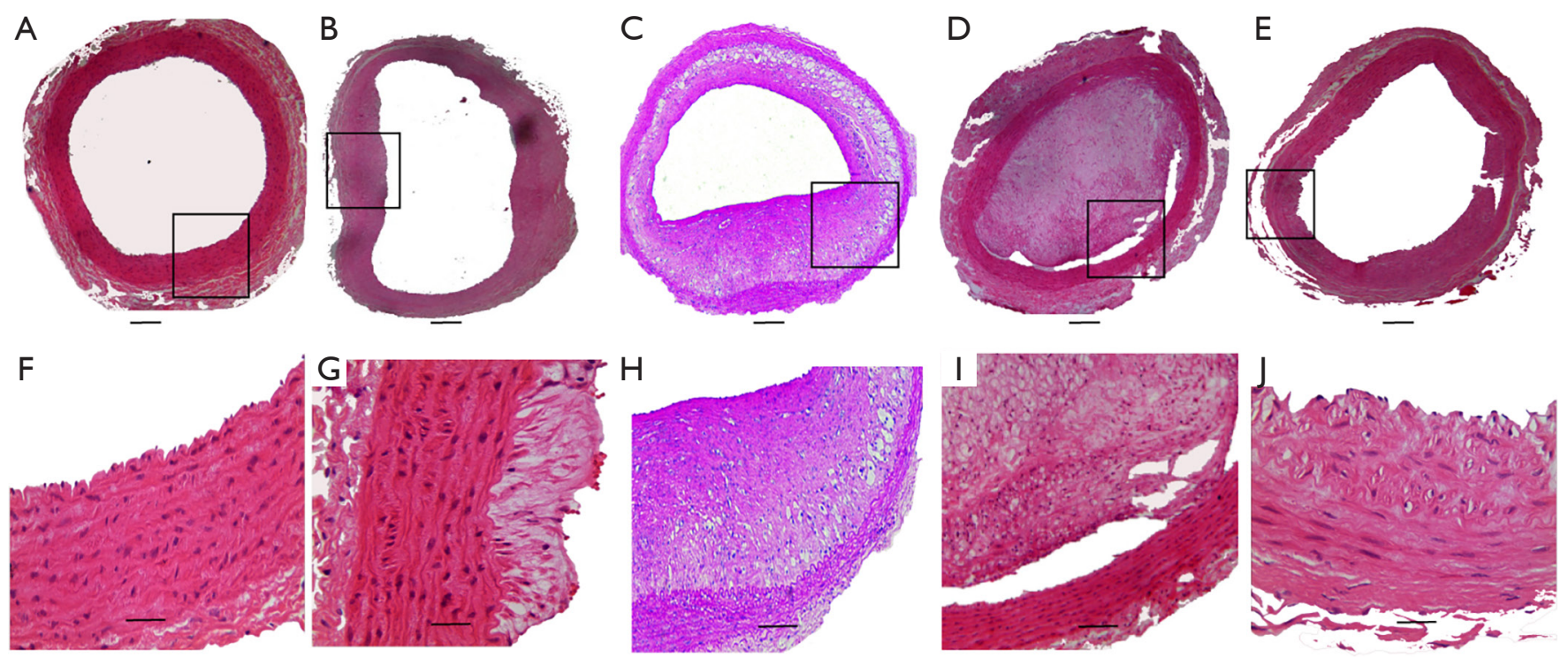

Figure 3 Morphological characteristics of atherosclerotic plaque stained with hematoxylin and eosin. Upper panels show a low-power view of the atherosclerotic plaque development in the carotid artery in rabbits with normal chaw diet (A), on Western-type diet (B), underwent balloon injury with Western-type diet (C), underwent dehydrated ethanol exposure with Western diet (D), and dehydrated ethanol exposure with normal chaw diet $(\mathrm{E})$, respectively. $\times 40$. (F-J) show a high-power view of the region outlined by the box in A, B, C, D and E, respectively $(\times 200)$. Scale bar $=200 \mu \mathrm{m}(\mathrm{A}-\mathrm{E})$ and $50 \mu \mathrm{m}(\mathrm{F}-\mathrm{J})$.

groups $\mathrm{E}$ and $\mathrm{B}$ were not significantly different $(\mathrm{P}>0.05)$.

\section{Characteristics of atherogenesis}

\section{Plaque burden}

The animals were killed when the observation period was completed, and right carotid artery specimens were collected to evaluate lesions. The characteristics of atherogenesis are summarized in Table 1 and Figure 3. The atherosclerotic plaque lesions were uniformly produced in all segments of the carotid artery that underwent dehydrated ethanol lavage (group D) and balloon injury (group C). Nevertheless, only fatty streak lesions were detected in the corresponding carotid arteries of animals in groups B and E. No evidence of atherosclerosis was noticed in the animals of group A. Additionally, the plaque burden in the vessels that underwent dehydrated ethanol lavage (group D) was more marked than that in those who underwent balloon injury (group C; 
$77 \% \pm 15 \%$ vs. $53 \% \pm 19 \% ; \mathrm{P}<0.01)$; hence, the MLA became significantly smaller $(0.51 \pm 0.20$ vs. $1.13 \pm 0.35 \mu \mathrm{m} ; \mathrm{P}<0.01)$, which was mainly ascribed to the higher MIT in the animals of group D $(613.5 \pm 110.6$ vs. $396.46 \pm 70.1 \mu \mathrm{m} ; \mathrm{P}<0.01$; Table 1). Moreover, most of the plaque burden in rabbits who underwent dehydrated ethanol lavage had an eccentric distribution, whereas the atherosclerotic lesions in the balloon-injury group were symmetrically distributed.

\section{Cellular composition}

To explore the nature of the plaques induced by dehydrated ethanol exposure, we further investigated the composition of vessel smooth muscle cells (VSMCs), ECs and macrophages, as well the expression of MMP-9 in the plaques (Figure 4). Compared with the normal vessels, those with atheroma induced by balloon injury (group C) and ethanol damage (group D) showed significantly increased and decreased $\mathrm{vWF}$ expression, respectively (both $\mathrm{P}<0.05)$. Moreover, the atheroma induced by dehydrated ethanol lavage showed a decreased expression of $\mathrm{vWF}$ and $\alpha-\mathrm{SMC}$, and more elevated levels of CD68 and MMP9 than did that induced by balloon injury (all $\mathrm{P}<0.01$ ). Hence, the cellular composition of the atheroma varied considerably in groups $\mathrm{C}$ and $\mathrm{D}$. These findings highlight the fact that atherosclerotic lesions induced by dehydrated ethanol exposure are more prone to vulnerability. We did not perform IHC study of related cellular components for groups B and E, as there was only fatty streak formation rather than progressive plaque in the carotid artery of rabbits fed with WD alone (group B) and injured by ethanol alone (group E).

\section{Evidence of plaque vulnerability}

Pathological geometry and cellular composition of atherosclerotic plaques in carotid arteries that were exposed to dehydrated ethanol were more likely to be vulnerable than those induced by balloon injury (Figures 3,4). Many atherosclerotic lesions in group D were characterized by subtotal occlusion and a large amount of macrophages accumulated in plaque (Figure 4). Of note, the minimal thickness of the fibrous cap in lesions induced by ethanol exposure were significantly thinner than that in lesions induced by balloon injury $(65.0 \pm 8.9$ vs. $109.2 \pm 22.5 \mu \mathrm{m}$; $\mathrm{P}<0.001$ ) (Figure 5). Moreover, large NC and intraplaque neovascularization were found in lesions induced by ethanol exposure (Figures 5,6). Taken together, these characteristics indicated the greater vulnerability of plaque induced by ethanol exposure.
Additionally, 3 of 6 rabbits in group D had ischemic stroke before specimen harvesting, and they displayed head tilt, disorientation, and motor disturbances with paralysis of 2 unilateral legs (Figure 6A). The atherosclerotic lesion responsible for the stroke was confirmed using CTA, indicating a subtotal blockage by the atheroma at the surgical segment, and a distal thrombotic occlusion in the vessel that underwent surgery (Figure 6B). The vessel specimen was harvested after the stroke events, and IHC staining illustrated the presence of a subtotal occlusive atheroma (advanced atherosclerotic plaque), multiple healed rupture sites, development of intraplaque neovascularization, and superimposed thrombosis [Figure 6(C1-C5)].

\section{Discussion}

In the present study, we successfully established a novel animal model for vulnerable atherosclerotic plaques using dehydrated ethanol lavage in the carotid arterial segment in rabbits fed a WD. The plaques induced by dehydrated ethanol lavage were characterized by a more severe plaque burden, a greater abundance of macrophages, a paucity of SMCs, higher MMP-9 expression in the plaque, and higher serum IL-1 $\beta$ and MCP-1 levels. Furthermore, animals who underwent ethanol treatment were more likely to experience ischemic stroke ascribed to the vulnerable carotid atherosclerotic lesions. Therefore, this animal model may represent a novel tool for studying the cardiovascular events associated with unstable atherosclerotic plaques in humans.

It is well known that endothelial injuries, regardless of cause, are critical to the development of human atherosclerotic lesions and subsequent cardiovascular events. Based on this principle, many animal models for the atherosclerotic lesions have been developed. Also, rabbits are still a powerful model for the study of human atherosclerosis (13), and the carotid artery would be an ideal vessel of interest. Before establishing the animal model, we performed an ex vivo experiment by investigating the growth curve with CCK-8 assay, which showed that the majority (90\%) of ECs died after 10 seconds of exposure to ethanol. Therefore, ethanol exposure should be an alternative approach model for EC injury, which may act as the trigger for the development of vulnerable atherosclerotic plaque. The lesion induced by dehydrated ethanol injury was then aggravated by the high-cholesterol diet. However, animals that underwent ethanol lavage and were fed a normal diet showed only endothelium denudation and fatty streaks instead of advanced atherosclerotic plaque, 
A

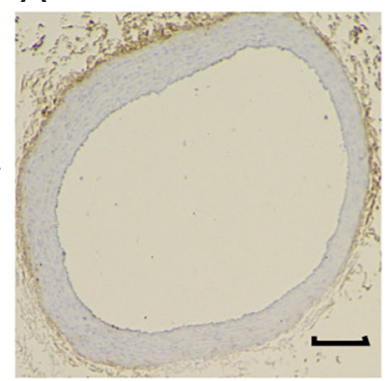

$\mathrm{E}$

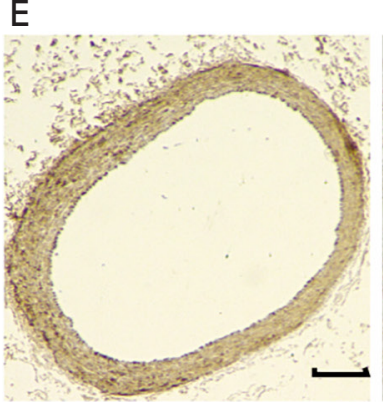

$\alpha-\mathrm{SMC}$

I

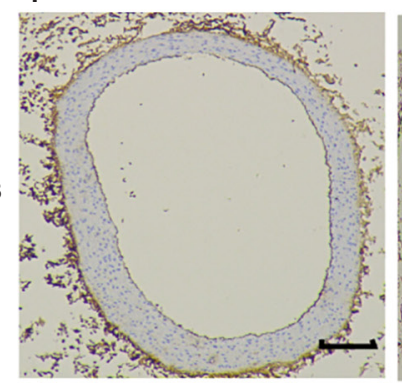

M

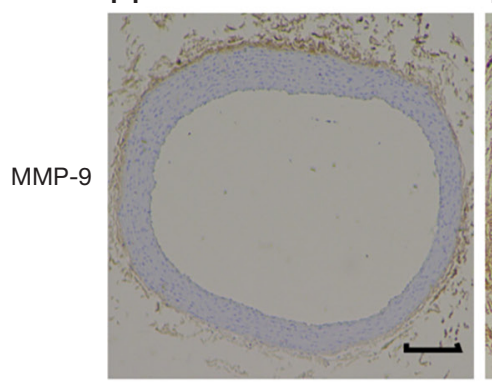

B

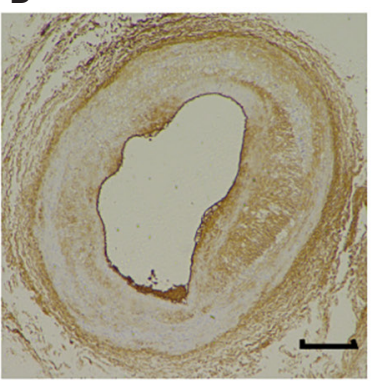

$\mathrm{F}$

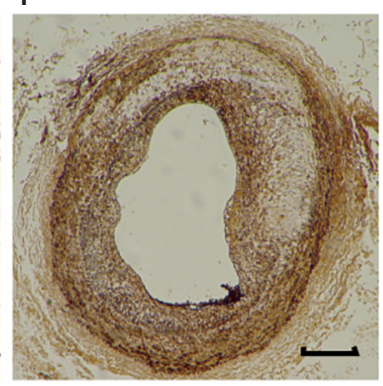

J

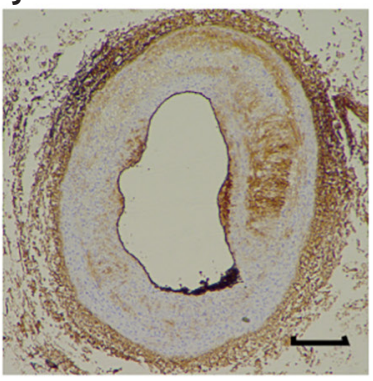

$\mathrm{N}$

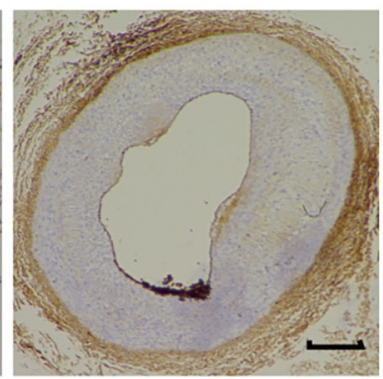

C

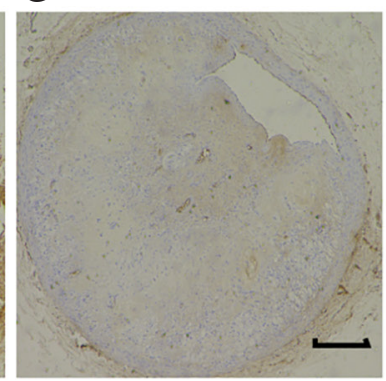

G

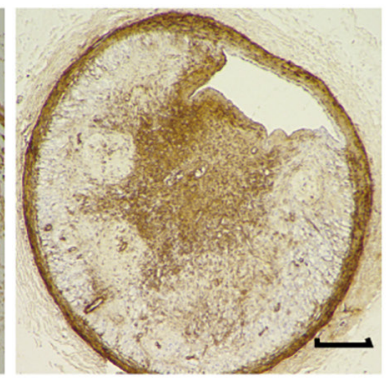

K

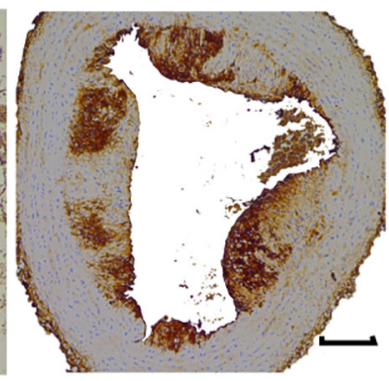

O

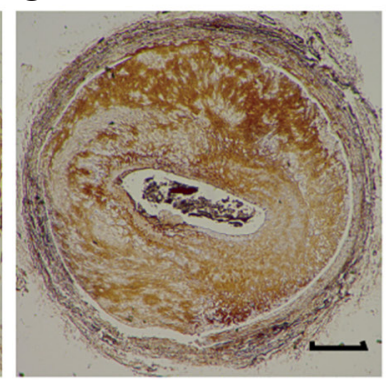

D

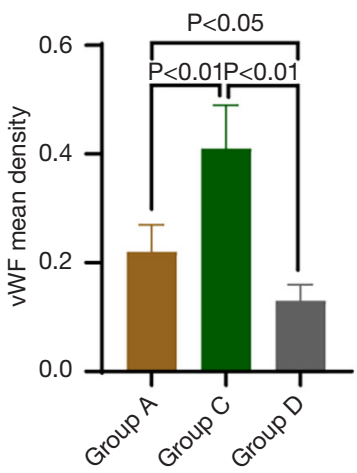

$\mathrm{H}$

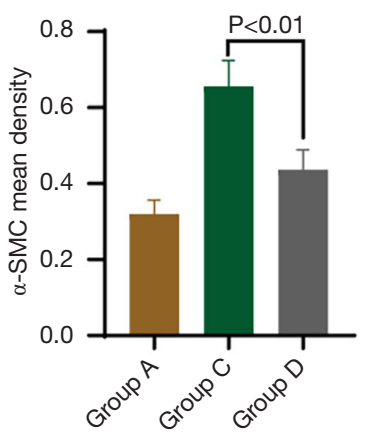

$\mathrm{L}$
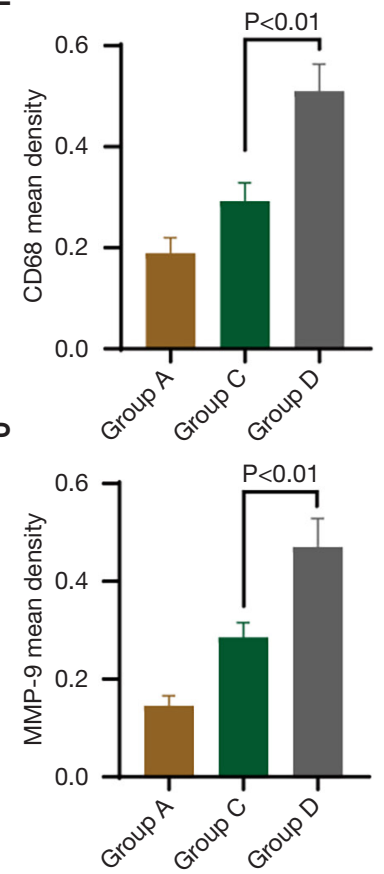

Figure 4 Cellular composition in the atherosclerotic plaque stained with immunohistochemical method. (A-C) show the presence of endothelial cells labeled by antibody against $\mathrm{vWF}$ in carotid arteries of rabbits fed a regular chow diet (group A), balloon injury followed by a Western diet (group C), and dehydrated ethanol exposure followed by a Western diet (group D), respectively (x40). (E-G) show the presence of smooth muscle cells labeled by antibody against $\alpha$-SMC in carotid arteries of rabbits in groups A, C, and D, respectively (x40). (I-K) show the presence of macrophages labeled by antibody against CD68 in carotid arteries of rabbits in groups A, C, and D, respectively (x40). (M-O) show the content of MMP-9 labeled by antibody against MMP-9 in carotid arteries of rabbits in groups A, C, and D, respectively $(\times 40)$. (D,H,L,P) show the results of statistical analysis (using the software Image-Pro Plus 6.0) for the mean density of staining against vWF, $\alpha$-SMC, CD68 and MMP-9 in carotid arteries, respectively. The dark red, green and grey columns in (D,H,L,P) represent the tissue staining density in rabbits of groups A, C and D, respectively. Comparisons were based on the data of 3 adjacent replicates stained with most significant atherosclerotic lesion per specimen ( $\mathrm{n}=18 /$ group). Carotid arteries from the rabbits fed a Western diet (group B) and undergone ethanol injury with normal chaw diet (group E) are not presented here, because only fatty streak rather than advanced atherosclerotic lesion was detected, for which immunohistochemical staining was not performed. Scale bar $=200 \mu \mathrm{m}$. vWF, von Willebrand factor; SMC, smooth muscle cell. 
A

B
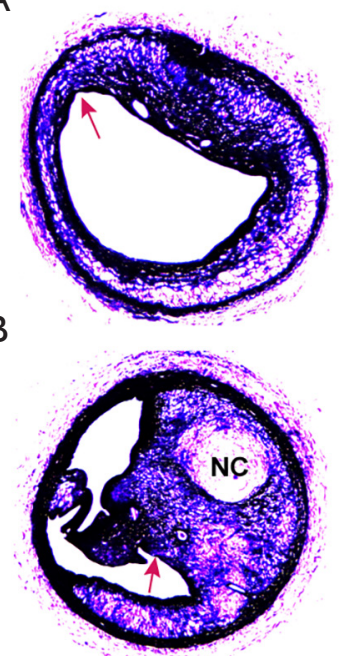

C

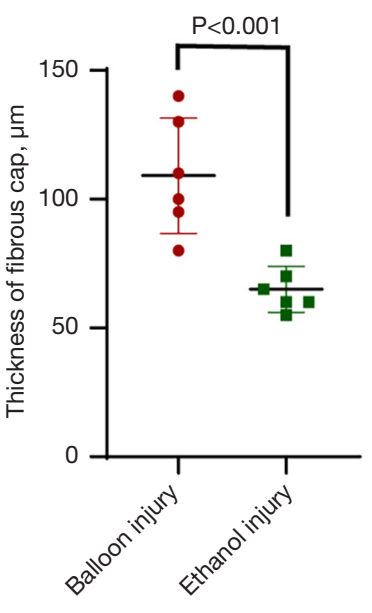

Figure 5 Fibrous cap thickness in relation to the injury type $(\mathrm{A}, \mathrm{B})$ show the fibrous cap thickness (indicated by red arrows) and plaque burden in atherosclerotic plaque induced by balloon injury with western-type $\operatorname{diet}$ (group C) and ethanol exposure with western-type diet (group D), respectively. (Movat pentachrome staining, $\times 40)$. The latter was characterized by more severe plaque load, thinner fibrous cap and large NC. Panel (C) illustrates the comparison of minimal thickness of fibrous cap in rabbits undergone balloon injury (group C) and ethanol exposure (group D) ( $\mathrm{n}=18$ replicates/group). Since the advanced atherosclerotic plaque did not develop in groups A, B and E, their data related to cap thickness was not provided here.

suggesting that typical advanced plaques must be dependent on the synergistic effect between ethanol damage and a highfat diet.

Comparing 2 models for atherogenesis, we found that the atherosclerotic lesions induced by balloon injury were relatively stable, manifesting as a thick fibrous cap, an abundance of SMCs, and less macrophage infiltration. In contrast, dehydrated ethanol lavage promoted the development of severe stenosis, which was usually complicated by plaque erosion, or rupture and subsequent formation of thrombosis, and eventually spontaneous stroke events. The progressive atherosclerotic plaque induced by dehydrated ethanol lavage was also characterized by an abundance of macrophages, a paucity of SMCs, and increased matrix degradation, the latter of which was indicated by a high MMP-9 content. Therefore, the higher vulnerability of atheroma was exhibited in the animal model that had undergone dehydrated ethanol lavage.

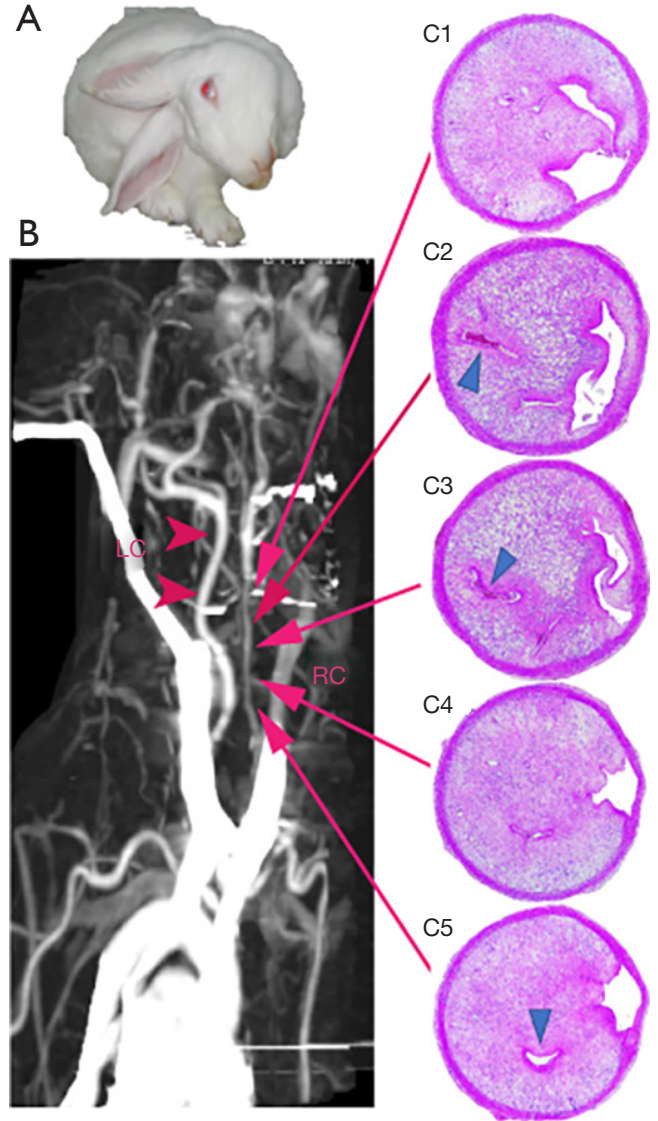

Figure 6 Angiographic and histological features of the atherosclerotic plaque in the rabbit undergone stroke event. (A) shows clinical signs of stroke, displaying head tilt and ipsilateral limb paralysis. (B) shows severe stenosis in the right carotid artery illustrated by CTA examination. (C1-C5) show the atherosclerotic lesions detected by histological staining (H\&E stain, $\times 40$ ) in the corresponding plane of (B) (indicated by arrows). The arrowheads in $(C)$ indicate the intra-plaque neovascularization occurred in lesions induced by ethanol exposure. RC and LC in panel B represent right and left carotid artery, respectively. Left carotid artery did not receive surgical procedure which shows normal manifestation. CTA, computer tomographic angiogram.

The majority of plaques in ethanol lavage rabbits were eccentrically distributed which can lead to increased tensile stress at the plaque borders and exacerbation of fissuring and thrombosis, and is thus predictive of vulnerable plaques (14). Moreover, thrombotic occlusion in the target carotid vessel, rather than acute brain injury resulting directly from ethanol, was likely responsible for the stroke events. We also noticed that advanced severe atherosclerotic plaque 
with intraplaque neovascularization occurred in lesions induced by ethanol exposure, which may be responsible the development of stroke events. Neovascularization in plaques leading to intraplaque bleeding and secondary thrombosis may be the possible reason for the occurrence of vascular events. Chen et al. successfully established an unstable atherosclerosis animal model by applying a tandem stenosis to the carotid artery of apolipoprotein E (ApoE)-deficient mice on a high-fat diet (15). They observed intraplaque hemorrhage in approximately $50 \%$ of mice. Later, they verified the identification of intraplaque hemorrhage with a novel technology, near-infrared autofluorescence imaging (16). Unfortunately, we did not use specific staining to further confirm the occurrence of intraplaque hemorrhage and neovascularization. Despite this limitation, cumulatively, these characteristics suggest that the lesions induced by ethanol injury tend to be more prone to rupture. This model is histologically similar to that in humans, being characterized by the formation of a typical fibrous cap, large $\mathrm{NC}$, and eventual occurrence of an ischemic stroke.

Cholesterol feeding and mechanical endothelial injury are 2 common features shared by most models of atherosclerosis. The rabbit model of plaque rupture was first presented by Rekhter et al. in 1998 (17), in which balloon catheters were used to mechanically injure the endothelium and thus produce a lesion in the thoracic aorta of cholesterol-fed rabbits. Some animal models of atherosclerosis have been developed using different approaches, such as immunologic or cold-induced endothelial injury $(18,19)$; however, none of the animal models of plaque rupture presented thus far can be regarded as ideal, as each has its own advantages and limitations with respect to the manipulation of the atherogenic process and the modeling of human atherosclerosis or lipoprotein profile $(20,21)$. Recently, a mouse model of plaque rupture was successfully generated by $\mathrm{ApoE}^{-/-}$and fibrillin $1(\mathrm{Fbn} 1)^{\mathrm{C} 1039 \mathrm{G}+/-}$ genetic mutation in combination with a WD (22), and by creating a tandem stenosis on the carotid artery of ApoE-deficient mice on a high-fat diet (15). However, all of these novel animal models are dependent on the special manipulation of a genetic defect. Therefore, they serve more as a proof for the role of a specific factor in the occurrence and development of atherosclerotic plaque instability, rather than as a suitable model for the investigation and application of a universal mechanism.

To ascertain whether localized carotid artery alcohol damage could cause systemic inflammation, we further examined circulating inflammatory markers including IL- $1 \beta$ and MCP-1. The marker IL- $1 \beta$ is secreted at high levels by macrophages, via activation of the NLRP3 inflammasome in response to atherogenic stimuli such as cholesterol crystals and therefore plays important roles in the initiation, progression, and rupture of atherosclerotic plaques (23-26). The chemokine MCP-1 regulates monocyte chemotaxis and T-lymphocyte differentiation, and also plays a crucial role in the pathogenesis of atherosclerosis (27-29). In this study, we found that IL-1 $\beta$ and MCP-1 were elevated more significantly in the dehydrated ethanol injury group than in the balloon injury group. It was revealed that measurement of hs-CRP could not distinguish between the atherogenesis induced by ethanol and that induced by balloon injury. This finding shows that serum IL- $1 \beta$ and MCP- 1 can more precisely predict the development of severe or vulnerable atherosclerotic plaque than can hs-CRP. However, the mechanism responsible for this finding and its clinical significance still warrant further investigation.

Additionally, this model has other advantages. Firstly, carotid arterial endothelial injury induced by a brief exposure to dehydrated ethanol mimics the biological trigger for the development of atherosclerosis in humans, which may be an improvement over mechanical maneuvers such as balloon injury and cold-induced injury, and better mimics the in vivo biological injury to the vessel endothelium. The degree and scope of the injury induced by dehydrated ethanol on the vessel tissues, including the endothelium, media, and even adventitia, are dose-(time)dependent, which can be easily controlled, evaluated, and replicated. However, the degree of vessel injury induced by balloon and cold is difficult to control. Finally, this method is inexpensive, simple, highly efficient, and reproducible, and is also less time-consuming (within 3 months) in successfully developing an animal model of vulnerable atherosclerotic plaque. However, it requires up to 14 months to develop a spontaneous plaque rupture in apoE knockout mice fed a high-fat diet $(30,31)$.

Nevertheless, there are still several limitations in the present animal model. Firstly, there is still no direct evidence for total occlusive thrombosis and fresh plaque disruption in this animal model. Secondly, this model also requires exogenous mechanical intervention for initiating the atherosclerotic lesions, which may be a common drawback in many animal models of atherosclerosis and unstable plaques. Thirdly, since we did not dynamically observe the formation and evolution of carotid atherosclerotic lesions, the observability of the early evolution and regression of plaque remains uncertain. Finally, there was a lack 
of staining for special components in atherosclerotic lesions, such as Oil Red $\mathrm{O}$ staining against foam cell, and identification of neovascularization and thrombosis components, whose inclusion will aid in increasing the understanding of these lesions' characteristics.

\section{Conclusions}

Overall, we successfully developed a novel animal model of vulnerable atherosclerotic plaques via dehydrated ethanol lavage in a carotid artery segment of rabbits maintained on a WD. This may represent a promising animal model for investigating new therapeutic targets and drugs, exploring new biomarkers, identifying novel imaging modalities for vulnerable plaques, and studying the mechanisms underlying the progress of atherosclerotic plaques.

\section{Acknowledgments}

The authors thanks to the Animal Laboratory, Ningbo University for the technical support and experiment assistance, to Dr. Chen Jie from Ningbo Pathology Diagnostic Center for her assistance and guidance in $\mathrm{HE}$ and IHC staining, and to Mr. J. Jones and J. Gray from AME editing service for their help in polishing the paper.

Funding: Zhejiang Provincial Natural Science Foundation, China (grant No. LY12H02001 to Cui H) and Zhejiang Provincial Key Research Project (grant No. 2021C03096 to Cui $\mathrm{H}$ ), as well as Ningbo Social Development Research Project (Grant No. 2011C51003 to Ye H).

\section{Footnote}

Reporting Checklist: The authors have completed the ARRIVE reporting checklist. Available at https://dx.doi. org/10.21037/cdt-21-291

Data Sharing Statement: Available at https://dx.doi. org/10.21037/cdt-21-291

Conflicts of Interest: All authors have completed the ICMJE uniform disclosure form (available at https://dx.doi. org/10.21037/cdt-21-291). HC reports that the study was supported by grant of Zhejiang Provincial Natural Science Foundation, China (grant No. LY12H02001) and Zhejiang Provincial Key Research Project (grant No. 2021C03096). HY reports that the study was partly supported by Ningbo Social Development Research Project (Grant No.
2011C51003 to HY). The other authors have no conflicts of interest to declare.

Ethical Statement: The authors are accountable for all aspects of the work in ensuring that questions related to the accuracy or integrity of any part of the work are appropriately investigated and resolved. Experiments were performed under a project license (NBU20210023) granted by institutional ethics committee board of Ningbo University, in compliance with Chinese national guidelines for the care and use of animals. A protocol was prepared before the study without registration.

Open Access Statement: This is an Open Access article distributed in accordance with the Creative Commons Attribution-NonCommercial-NoDerivs 4.0 International License (CC BY-NC-ND 4.0), which permits the noncommercial replication and distribution of the article with the strict proviso that no changes or edits are made and the original work is properly cited (including links to both the formal publication through the relevant DOI and the license). See: https://creativecommons.org/licenses/by-nc-nd/4.0/.

\section{References}

1. Roger VL, Go AS, Lloyd-Jones DM, et al. Heart disease and stroke statistics--2012 update: a report from the American Heart Association. Circulation 2012;125:e2-e220.

2. Hansson GK, Heistad DD. Two views on plaque rupture. Arterioscler Thromb Vasc Biol 2007;27:697.

3. Libby P. Current concepts of the pathogenesis of the acute coronary syndromes. Circulation 2001;104:365-72.

4. Libby P, Ridker PM, Hansson GK. Progress and challenges in translating the biology of atherosclerosis. Nature 2011;473:317-25.

5. Silvestre-Roig C, de Winther MP, Weber C, et al. Atherosclerotic plaque destabilization: mechanisms, models, and therapeutic strategies. Circ Res 2014;114:214-26.

6. Cullen P, Baetta R, Bellosta S, et al. Rupture of the atherosclerotic plaque: does a good animal model exist? Arterioscler Thromb Vasc Biol 2003;23:535-42.

7. Massaro M, Scoditti E, Carluccio MA, et al. Alcohol and atherosclerosis: a double edged sword. Vascul Pharmacol 2012;57:65-8.

8. Shirpoor A, Salami S, Khadem-Ansari MH, et al. Longterm ethanol consumption initiates atherosclerosis in rat aorta through inflammatory stress and endothelial dysfunction. Vascul Pharmacol 2012;57:72-7. 
9. Hess OM, Sigwart U. New treatment strategies for hypertrophic obstructive cardiomyopathy: alcohol ablation of the septum: the new gold standard? J Am Coll Cardiol 2004;44:2054-5.

10. Waxman S, Ishibashi F, Muller JE. Detection and treatment of vulnerable plaques and vulnerable patients: novel approaches to prevention of coronary events. Circulation 2006;114:2390-411.

11. Bennett MR. Breaking the plaque: evidence for plaque rupture in animal models of atherosclerosis. Arterioscler Thromb Vasc Biol 2002;22:713-4.

12. Virmani R, Kolodgie FD, Burke AP, et al. Lessons from sudden coronary death: a comprehensive morphological classification scheme for atherosclerotic lesions. Arterioscler Thromb Vasc Biol 2000;20:1262-75.

13. Fan J, Chen Y, Yan H, et al. Principles and Applications of Rabbit Models for Atherosclerosis Research. J Atheroscler Thromb 2018;25:213-20.

14. Slager CJ, Wentzel JJ, Gijsen FJ, et al. The role of shear stress in the destabilization of vulnerable plaques and related therapeutic implications. Nat Clin Pract Cardiovasc Med 2005;2:456-64.

15. Chen YC, Bui AV, Diesch J, et al. A novel mouse model of atherosclerotic plaque instability for drug testing and mechanistic/therapeutic discoveries using gene and microRNA expression profiling. Circ Res 2013;113:252-65.

16. Htun NM, Chen YC, Lim B, et al. Near-infrared autofluorescence induced by intraplaque hemorrhage and heme degradation as marker for high-risk atherosclerotic plaques. Nat Commun 2017;8:75.

17. Rekhter MD, Hicks GW, Brammer DW, et al. Animal model that mimics atherosclerotic plaque rupture. Circ Res 1998;83:705-13.

18. Zhang G, Li M, Li L, et al. The immunologic injury composite with balloon injury leads to dyslipidemia: a robust rabbit model of human atherosclerosis and vulnerable plaque. J Biomed Biotechnol 2012;2012:249129.

19. Fang SM, Zhang QH, Jiang ZX. Developing a novel rabbit model of atherosclerotic plaque rupture and thrombosis by cold-induced endothelial injury. J Biomed Sci 2009;16:39.

20. Xiangdong L, Yuanwu L, Hua Z, et al. Animal models for the atherosclerosis research: a review. Protein Cell 2011;2:189-201.

21. Getz GS, Reardon CA. Animal models of atherosclerosis. Arterioscler Thromb Vasc Biol 2012;32:1104-15.
22. Van der Donckt C, Van Herck JL, Schrijvers DM, et al. Elastin fragmentation in atherosclerotic mice leads to intraplaque neovascularization, plaque rupture, myocardial infarction, stroke, and sudden death. Eur Heart J 2015;36:1049-58.

23. Crea F, Liuzzo G. Addressing Acute Coronary Syndromes: New Challenges and Opportunities After the CANTOS Trial (Canakinumab Anti-inflammatory Thrombosis Outcomes Study). Circulation 2018;137:1100-2.

24. Libby P, Ridker PM, Hansson GK, et al. Inflammation in atherosclerosis: from pathophysiology to practice. J Am Coll Cardiol 2009;54:2129-38.

25. Duewell P, Kono H, Rayner KJ, et al. NLRP3 inflammasomes are required for atherogenesis and activated by cholesterol crystals. Nature 2010;464:1357-61.

26. Kirii H, Niwa T, Yamada Y, et al. Lack of interleukin-1beta decreases the severity of atherosclerosis in ApoE-deficient mice. Arterioscler Thromb Vasc Biol 2003;23:656-60.

27. Gerszten RE, Garcia-Zepeda EA, Lim YC, et al. MCP1 and IL-8 trigger firm adhesion of monocytes to vascular endothelium under flow conditions. Nature 1999;398:718-23.

28. Ohman MK, Wright AP, Wickenheiser KJ, et al. Monocyte chemoattractant protein-1 deficiency protects against visceral fat-induced atherosclerosis. Arterioscler Thromb Vasc Biol 2010;30:1151-8.

29. Lutgens E, Faber B, Schapira K, et al. Gene profiling in atherosclerosis reveals a key role for small inducible cytokines: validation using a novel monocyte chemoattractant protein monoclonal antibody. Circulation 2005;111:3443-52.

30. Johnson JL, Jackson CL. Atherosclerotic plaque rupture in the apolipoprotein E knockout mouse. Atherosclerosis 2001;154:399-406.

31. Bond AR, Jackson CL. The fat-fed apolipoprotein E knockout mouse brachiocephalic artery in the study of atherosclerotic plaque rupture. J Biomed Biotechnol 2011;2011:379069.

Cite this article as: Zhao $\mathrm{R}$, Liu $\mathrm{H}$, Zhang S, Lu Q, Fei X, Zhou H, Liu J, Ye H, Chen X, Cui H. A novel animal model for vulnerable atherosclerotic plaque: dehydrated ethanol lavage in the carotid artery of rabbits fed a Western diet. Cardiovasc Diagn Ther 2021;11(6):1241-1252. doi: 10.21037/cdt-21-291 